\title{
PROGRESS ON THE SECTOR MAGNETS FOR THE RIKEN SRC
}

\author{
A. Goto, H. Okuno, J. Ohnishi, N. Fukunishi, T. Mitsumoto, S. Fujishima, T. Tominaka, \\ K. Ikegami, Y. Miyazawa, and Y. Yano, RIKEN, Wako, Saitama 351-0198, Japan
}

\begin{abstract}
Significant changes have been made to the design of the sector magnet of the RIKEN SRC (superconducting ring cyclotron). The changes are: (1) the cyclotron is almost completely covered with soft-iron slabs of $0.8 \mathrm{~m}$ in thickness, except in the central region, to reduce stray fields, and (2) the cold-pole scheme has been replaced with a warm-pole scheme. Owing to this magnetic shield, the stray fields, magneto-motive forces, electromagnetic forces, and so on are significantly reduced. New design of the real sector magnet is described in this report.
\end{abstract}

\section{INTRODUCTION}

A K2500-MeV Superconducting Ring Cyclotron (SRC) is under construction for the RIKEN RI Beam Factory[1]. At the last conference of this series we reported on the design of the sector magnet as well as the status of a prototype sector magnet[2]. The design of the sector magnet, however, was changed significantly before starting to fabricate the real ones so as mainly to reduce the stray field from the cyclotron. We thus quitted further fabrication of the prototype sector magnet, and have concentrated our effort on the new design. The new design has almost been finalized.

\section{CHANGES TO THE FUNDAMENTAL DESIGN}

The changes of the design from the prototype sector magnet to the real one are as follows: (1) A pair of large active magnetic-shield coils has been replaced with soft iron slabs that cover the valley regions, and (2) the coldpole scheme has been replaced with a warm-pole scheme.

In the new design, the cyclotron is almost completely covered with soft-iron slabs of $0.8 \mathrm{~m}$ in thickness, except in the central region. The "iron cover" yields the following good results:

(1) The SRC can be self-radiation-shielding.

(2) The stray field in the valley is reduced from $0.5 \mathrm{~T}$ to $0.1 \mathrm{~T}$ at the maximum. The sector field needed to bend, for example, $\mathrm{U}^{58+} 150 \mathrm{MeV} /$ nucleon is accordingly reduced from $4.3 \mathrm{~T}$ to $3.7 \mathrm{~T}$ because of this reduction of negative stray field, with the K-value of the SRC being kept at 2,500 MeV. According to this lower flutter, we relocate the operating domain of the vertical betatron frequencies from the values between 1.0-1.5 to those between 0.5-1.0. The decrease of the vertical betatron frequencies causes an increase in the vertical beam size by a factor of about 1.4 , but this presents no problem because of sufficient acceptance of the injection and extraction elements. This decrease also makes the tolerances of the main and trim coils and poles tighter, but they are still tolerable. According to the reduction of the maximum sector field, the maximum magneto-motive force required is also reduced from 5.4 MA/sector to about 3.8 MA/sector. The maximum stored energy and the electromagnetic forces exerted on the main coil are greatly reduced from $390 \mathrm{MJ}$ to $235 \mathrm{MJ}$ and from $400 \mathrm{Ton} / \mathrm{m}$ to 260 Ton $/ \mathrm{m}$, respectively. This reduction of forces allows us to adopt a warm-pole scheme; there is now no concern about brittleness of the iron pole at low temperature. The small stray field in the valley allows us to use cryopumps, motors, control devices, and so on in a safe situation.

(3) Superconducting magnetic channels for beam injection and extraction are unnecessary. Now all the injection and extraction magnetic channels inside gap of the sector magnets are normal with the moderate power consumption, and their structures are very similar to those for the RRC and the IRC[3].

(4) The shift of the injection and extraction trajectory depending on the negative stray field strength is greatly reduced (almost no shift).

(5) The stray field outside the SRC is reduced to about 200 gauss at the maximum near the yoke and the vertical side shield-wall. We need neither the active magnetic shielding which was adopted in the old design nor the thick iron plates enclosing the huge SRC vault. We place the rf oscillators near the SRC like they are for the RRC and the IRC. The SRC vault is now very safe for those working at the site even inside the cyclotron.

\section{MECHANICAL STRUCTURE AND CHARACTERISTICS}

\subsection{General Features}

A layout of the SRC is shown in Fig. 1. The SRC consists mainly of six sector magnets, four main rf resonators, a flattop rf resonator, injection and extraction elements. The valley regions are covered with the magnetic shield irons. The $\mathrm{K}$-value is $2,500 \mathrm{MeV}$. The outer radius and height of the SRC are $9.2 \mathrm{~m}$ and $7.6 \mathrm{~m}$, respectively. The mean injection and extraction radii are $3.56 \mathrm{~m}$ and 5.36 $\mathrm{m}$, respectively. The SRC allows us to accelerate light heavy-ions at $400 \mathrm{MeV} /$ nucleon and very heavy ions even at $350 \mathrm{MeV} /$ nucleon[1]. 


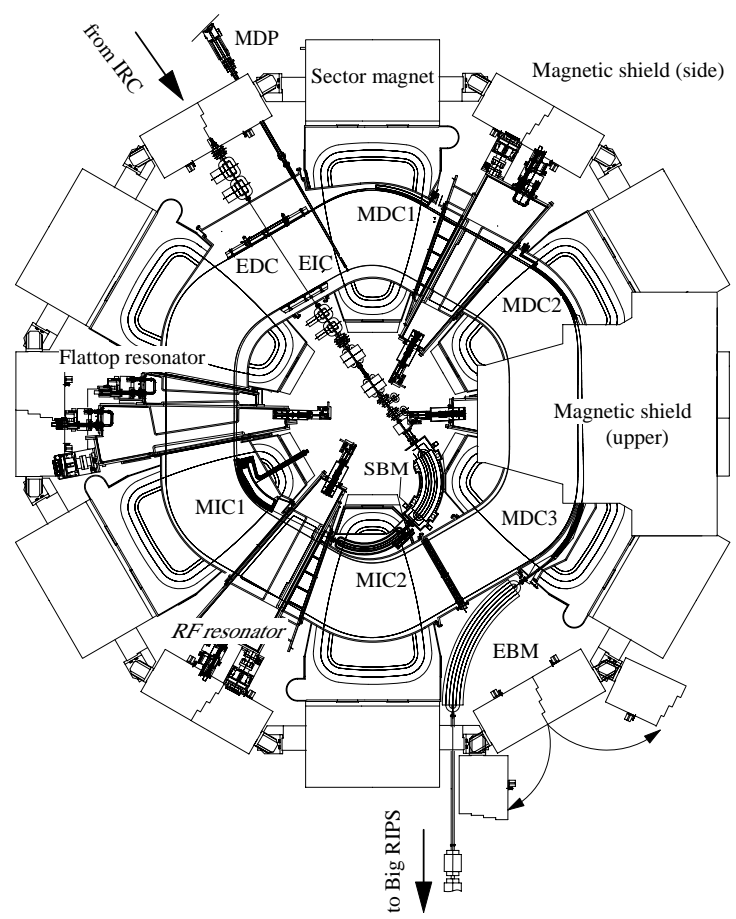

Figure 1: Plan view of the SRC.
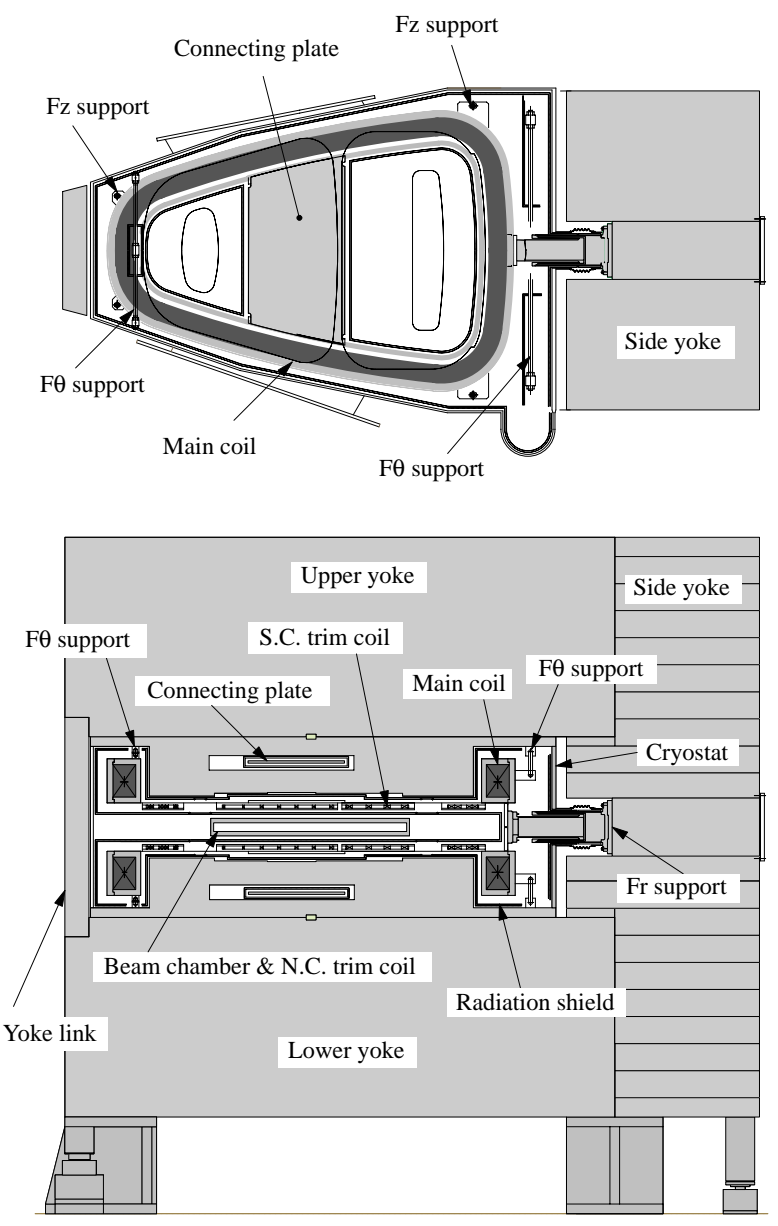

Figure 2: Cross-sectional and plan views of the sector magnet.

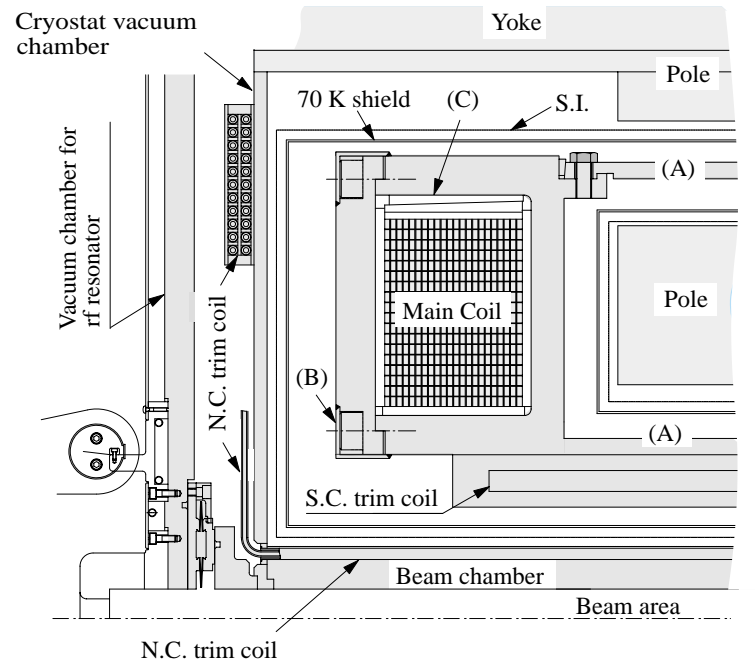

$\begin{array}{lll}\text { (A) : Connecting plate } & \text { (B) : Seal cover } & \text { (C) : Cotta }\end{array}$

Figure 3: Cross-sectional view of the main coil, superconducting trim coils, pole, yoke and others.

Cross-sectional and plan views of the newly designed sector magnet (excluding the magnetic shields) are shown in Fig. 2. The sector magnet is $7.2 \mathrm{~m}$ in length and $6 \mathrm{~m}$ in height. The weight is about 800 Ton per each. The sector angle is $25 \mathrm{deg}$. The maximum sector field is $3.8 \mathrm{~T}$, which is required to accelerate $350 \mathrm{MeV} /$ nucleon $\mathrm{U}^{88+}$ ions. Main components of the sector magnet are: a pair of superconducting main coils, four sets of superconducting trim coils, their cryostat, thermal insulation support links, twenty-two pairs of normal conducting trim coils, warmpoles and a yoke. Figure 3 shows a cross-sectional view of these components.

\subsection{Main Coil}

According to the reduction of the required magnetomotive force, the total number of turns per coil has been reduced from 600 to 396: 22 layers (horizontal) times 18 turns (vertical). The other structures are designed based on the old design.

The maximum current of the main coil is 5,000 A, giving the maximum magneto-motive force per sector of 4.0 MA. A solenoid winding is adopted for the main coil with cooling gaps of $0.8 \mathrm{~mm}$ and $1.5 \mathrm{~mm}$ horizontally and vertically, respectively. Spacers made of FRP (Fiber Reinforced Plastic) are placed in the both gaps; about $50 \%$ of the vertical conductor surface are exposed to liquid helium, while no horizontal surface is exposed in order to increase the reliability of electric insulation. Crosssectional area of the coil measures $208 \mathrm{~mm}$ by $284 \mathrm{~mm}$. The maximum current density is $34 \mathrm{~A} / \mathrm{mm}^{2}$. The main coil is designed based on Maddock's partial stabilization criterion.

The superconductor has a rectangular shape consisting of a Rutherford-type NbTi cable located at the center of conductor and a stabilizer housing[2]. The conductors' 
cross-sectional area measures $8 \mathrm{~mm}$ by $15 \mathrm{~mm}$. The stabilizer material is aluminum alloy with $1000 \mathrm{ppm} \mathrm{Ni}$, which gives a high $0.2 \%$-yield strength of about $60 \mathrm{MPa}$ at room temperature. The residual resistivity ratio is greater than 800. Cryogenic stabilized current at $6 \mathrm{~T}$ estimated from the measured resistivity and heat flux is greater than $6,000 \mathrm{~A}$. Total length of the conductors for the six sector magnets is $51 \mathrm{~km}$.

The main coil vessel is made of stainless steel. The thickness is $50 \mathrm{~mm}$ or $60 \mathrm{~mm}$. The C-shaped vessel is made at first, then the coil winding is performed with a tension stress of $20 \mathrm{MPa}$, the outer plate is attached with screws made of high-strength stainless steel (A286), and finally thin L-shaped seal covers are welded to the vessel. Pre-stress to the coil is put at a pressure of $10 \mathrm{MPa}$ with a cotta and the outer plate vertically and horizontally, respectively. The vessel is designed in such a way that there is no straight section for the coil winding.

The horizontal electromagnetic force of about 260 Ton $/ \mathrm{m}$ is exerted on the long section of the vessel covering the beam area. In order to sustain this force, a pair of connecting plates is attached to the both sides of this section. The plate is $1 \mathrm{~m}$ in width and $25 \mathrm{~mm}$ in thickness. One of these plates crosses through the rectangular hole (1.5 $\mathrm{m}$ in width and $160 \mathrm{~mm}$ in height) of the warm iron pole. The stress on these plates is about $90 \mathrm{MPa}$ and that on the seat of the vessel for the connecting plate is about $500 \mathrm{MPa}$. The maximum deformation of the vessel due to electromagnetic forces is $1.8 \mathrm{~mm}$ with the maximum stress on the vessel of $230 \mathrm{MPa}$. Although the mechanical stiffness of this structure decreases compared with that of the cold-pole structure in the old design, it is still rigid enough to support the electromagnetic forces owing to the reduction of the magnetic motive forces.

\subsection{Superconducting and Normal-conducting Trim Coils}

Two types of trim coils are used to help generate isochronous fields for various kinds of ions: superconducting trim coils and normal-conducting trim coils.

Superconducting trim coils consist of four sets (parameters) as shown in Fig. 4. In the new design a doublepancake winding is adopted, in which the coils are indirectly cooled by forced two-phase helium through tubes engraved on the coil case as shown in Fig. 5. (In the old design solenoid windings using a superconducting wire of $2.9 \mathrm{~mm} \times 3.6 \mathrm{~mm}$ in size were bath-cooled in a He vessel). This design change was made because there had been a concern about the exhaustion of helium gas from the $\mathrm{He}$ vessel in the old design. The same super-conductor as that for the main coil is used. The four sets of trim coils consist of 2 (layers) x 4 (turns) x 2 (blocks), 2 × 5 × 5,2 × 7 x 2 and $2 \times 8 \times 2$, respectively. The total length of the conductors for the six sector magnets is $7 \mathrm{~km}$. The maximum current is 3,000 A. The coil case is made of two aluminum-alloy plates of $18 \mathrm{~mm}$ in thickness per each that

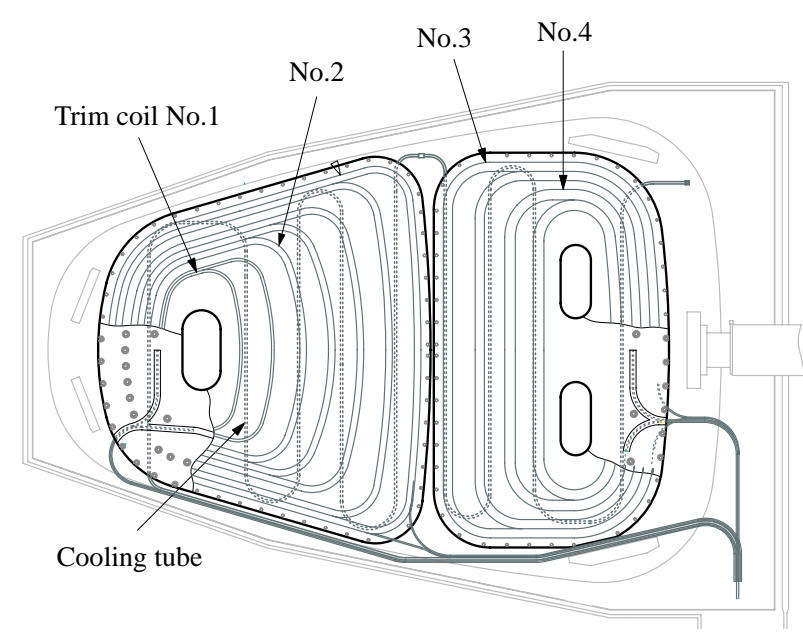

Figure 4: Plan view of the superconducting trim coils.

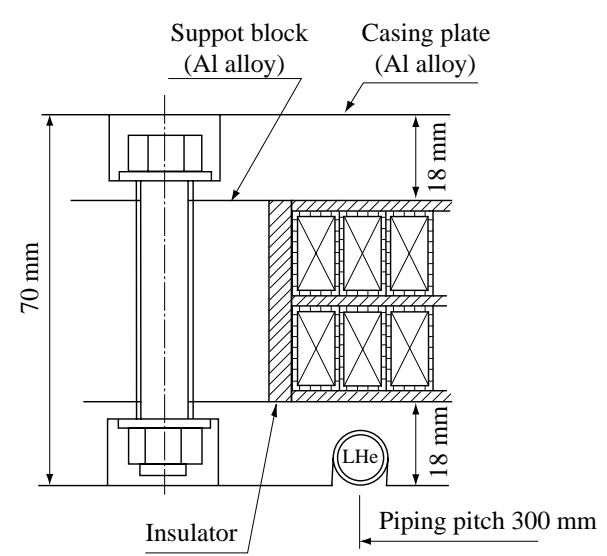

Figure 5: Cross-sectional view of the superconducting trim coils.

sandwich the trim coils and support blocks with screws. The liquid-helium cooling tubes are arranged with a pitch of $300 \mathrm{~mm}$. For the insulation of the coils, the superconductor is wound with about $80 \mu \mathrm{m}$-thick adhesive bonding tape with epoxy. And then about $0.2 \mathrm{~mm}$ thick GUG (or GKG) film together with about $0.3 \mathrm{~mm}$-thick adhesive bonding FRP plate are inserted both between adjacent superconductors and between the superconductors and the coil case. The coil case is divided into two parts, each containing two of the four sets. The maximum deformation of the case due to electromagnetic forces is about 0.6 $\mathrm{mm}$ and the maximum bending stress is about $20 \mathrm{MPa}$. These coil cases are attached to the main coil vessel with screws.

Twenty-two pairs of normal-conducting trim coils are attached on the surface of the beam chamber that is part of the cryostat, as shown in Fig. 6. The maximum current is $600 \mathrm{~A}$. The coil is made of a double-tube consisting of a copper conductor of $10 \mathrm{~mm}$ in diameter covered with a stainless steel of $17 \mathrm{~mm}$ in diameter with an insulator in between. 


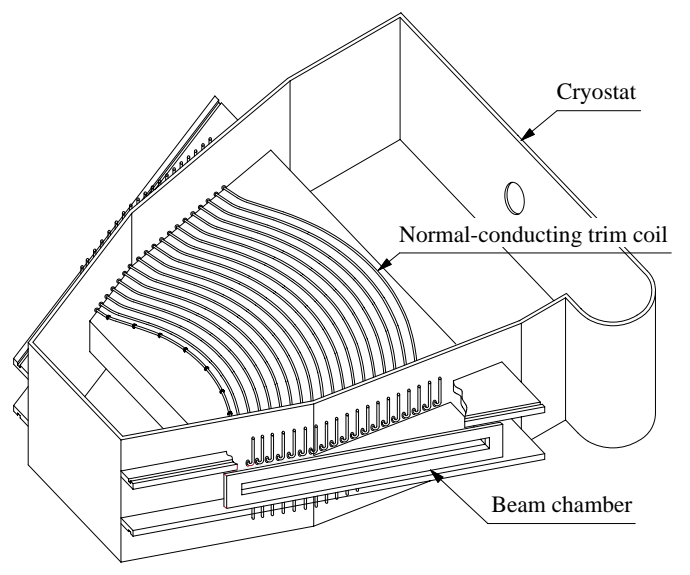

Figure 6: Conceptual drawing of the cryostat, beam chamber and normal-conducting trim coils.

\subsection{Cryostat and Beam Chamber}

Conceptual drawing of the cryostat and beam chamber is shown in Fig. 6 (the upper and lower walls are not shown in this figure). The side walls of the cryostat are made of stainless steel. The upper and lower walls, which constitute part of pole, are made of steel. The widths of the side walls are $20 \mathrm{~mm}, 30 \mathrm{~mm}$ and $40 \mathrm{~mm}$ for the connection part faced with the resonator or valley chamber, for the inner-radius part and the outer radius part, respectively. The width of the upper and lower walls is $30 \mathrm{~mm}$. The side walls and the upper and lower walls are welded to each other. The maximum deformation of the walls due to atmospheric pressure is $5 \mathrm{~mm}$. The maximum stress at the welded part is $100 \mathrm{MPa}$. The distances from the $4.5 \mathrm{~K}$ vessel are more than $95 \mathrm{~mm}$ for the side walls, more than $65 \mathrm{~mm}$ for the warm pole and $130 \mathrm{~mm}$ for the upper and lower walls, respectively. The gap distance between the connecting plate and the surface of the hole of the warm pole is $70 \mathrm{~mm}$. These gaps are wide enough to incorporate $70 \mathrm{~K}$ thermal radiation shields. The area of the radiation shields is about $85 \mathrm{~m}^{2}$. The thickness of the beam chamber wall made of stainless steel is $40 \mathrm{~mm}$, and the gap of the chamber is $90 \mathrm{~mm}$ in which the injection and extraction elements are placed.

\subsection{Thermal-Insulation Support Link}

The cold mass is supported with a total of 17 thermalinsulation support links as shown in Fig. 7. A multicylinder type of support link is used for the radial support. The multi-cylinder is made of stainless steel with an outermost diameter of $320 \mathrm{~mm}$ and a length of $850 \mathrm{~mm}$. It is designed to sustain the radial shifting force of 90 Ton. It is fixed on the surface of the back yoke. The vertical (azimuthal) support is performed with four (also four) support links at inner-radius part and four (also four) at outer-radius part are used for the vertical (azimuthal) direction. They are made of titanium-alloy rod with a diameter of $36 / 42(28 / 34) \mathrm{mm}$, and are designed to have a spring constant with larger than 140/190 (100/85) kN/mm

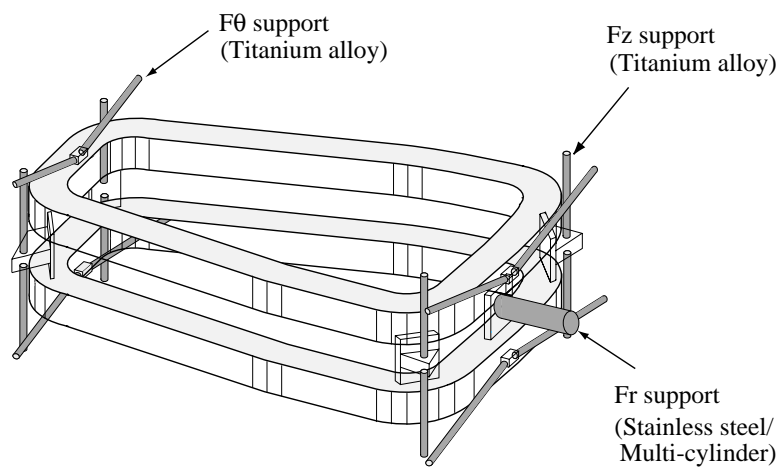

Figure 7: Conceptual drawing of the thermal-insulation support links.

to sustain the vertical (azimuthal) unbalanced force. The vertical support links are fixed at the upper and lower yokes. The azimuthal links are fixed on the surface of the upper and lower walls of the cryostat. The whole support links are designed to sustain the additional force due to earthquake of 1,000 Gal and $500 \mathrm{Gal}$ in the horizontal and vertical directions, respectively.

\subsection{Pole and Yoke}

The pole is divided into two pieces in order to make a hole that lets the connecting plate of the main coil vessel cross through it, as shown in Fig. 8. These poles are fixed to the surface of the upper/lower yoke with long screws through the yoke. The vacuum seals are made by welding the surface of the pole 2 and the edges of the holes (for these screws) of the upper/lower cryostat walls. The pole 1 is attached to the pole 2 with screws from the median plane side. A total force of 760 Ton due to electromagnetic force and atmospheric pressure is exerted on a pair of poles including the cryostat wall toward the median plane. The area of aperture of the hole measures $160 \mathrm{~mm}$ by $1,500 \mathrm{~mm}$. The pole gap is stepwise: 640,700 and 714 $\mathrm{mm}$. The sizes of the hole and the gap are optimized to produce various isochronous fields effectively. The weight of the pole per sector is about 60 Ton.

The slabs of the upper and lower yokes are stacked in the horizontal direction in order to keep the deformation due to electromagnetic forces as small as possible. The typical slab is $5.7 \mathrm{~m}$ (length) $\times 0.3 \mathrm{~m}$ (thickness) $\times 2.2 \mathrm{~m}$ (width). The weight of the yoke per sector is about 750 Ton.

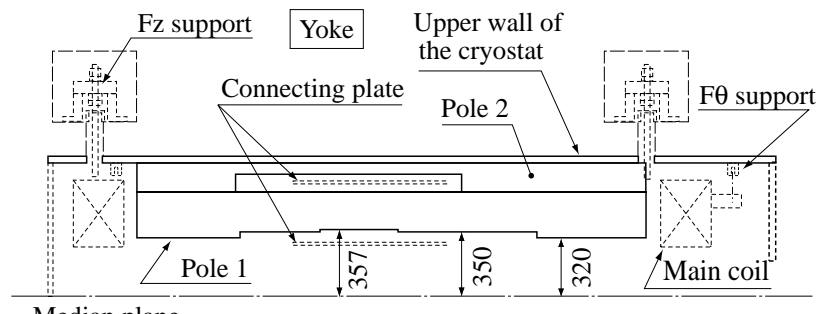

Median plane

Figure 8: Cross-sectional view of the pole. 


\subsection{Magnetic Shield}

Some of the iron slabs of the magnetic shield are bridged on the top and bottom of the valley regions between the sector magnets. The others are placed vertically between these top and bottom slabs so as to cover the space between the back yokes of the neighboring sector magnets. Cross-sectional and plan views of the magnetic shield are shown in Fig. 9. The total weight of these six falling-U-shaped structures is about 3,000 Ton (the total weight of the six sector magnets amounts to 7,800 Ton). The vertical outside slabs are assembled to form a double-

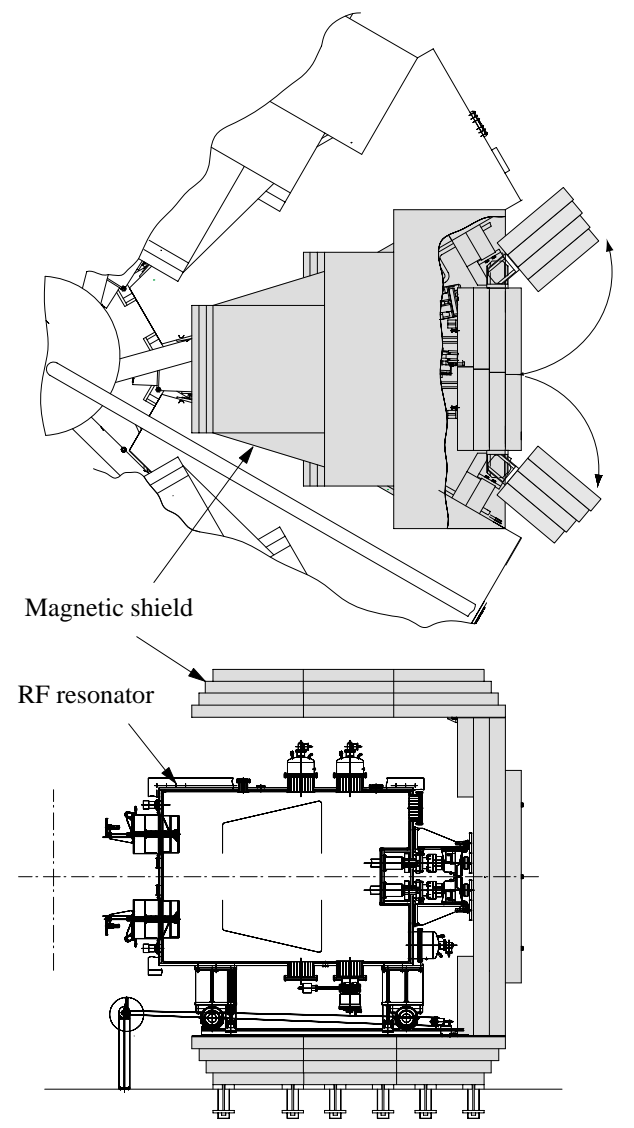

Figure 9: Cross-sectional and plan views of the magnetic shield.

leafed hinged door to be opened when the maintenance is carried out for the rf resonators, vacuum pumps, and so on in the valley region.

\section{QUENCH PROTECTION SYSTEM}

In order to protect the superconducting main and trim coils, the quench characteristics was calculated in terms of current decay, temperature rise, voltage development. In this simulation, the effects of the nonlinear inductance due to the iron pole and yoke of each sector, the mutual coupling between the main and trim coils, and the eddy current induced on the aluminum case of trim coils were taken into account.
The simulation shows that the optimal resistance of dump resistor should be $0.3 \Omega$ and $0.1 \Omega$ for the main coil and the trim coil, respectively. The temperature of the main coil rises up to about $140 \mathrm{~K}$ with this dump resistor. The maximum voltage applied between the main coil and the coil vessel can be half of $1,500 \mathrm{~V}$, by taking the earth at the middle point of the dump resistor.

\section{CRYOGENIC COOLING SYSTEM}

The cooling system is a closed-circuit system without liquid nitrogen. It consists mainly of the six cryostats of the sector magnets, the cryostat of the injection bending magnet, a control dewar, a refrigerator, three compressors and five buffer tanks. The control dewar of $2.5 \mathrm{~m}$ in diameter and $3 \mathrm{~m}$ in height is located on the top of the six sector magnets. The superconducting conductors of the coils are electrically connected to the power leads in the liquid helium vessel of the control dewar. The total volume of liquid helium in the main coil vessels for the six sector magnets is about $2,100 \mathrm{~L}$ and that in the control dewar is 2,000 L.

By taking the injection bending magnet[4] into account, the refrigerator is designed to have a capacity of $600 \mathrm{~W}$ at $4.5 \mathrm{~K}$ for the coils, $3500 \mathrm{~W}$ at $70 \mathrm{~K}$ for the thermal shields, and $4 \mathrm{~g} / \mathrm{s}$ for the current leads. The total equivalent refrigeration capacity at $4.5 \mathrm{~K}$ is thus about $1.3 \mathrm{~kW}$.

The time required to cool-down the cold mass of 180 Ton in total (110 Ton for the stainless steel vessels and 70 Ton for the aluminum super-conductors and casing plates for the superconducting trim coils) from room temperature to $4.5 \mathrm{~K}$ is estimated to be about 20 days.

\section{SUMMARY}

Significant changes have been made to the design of the sector magnet. By covering the cyclotron with magnetic shields, the stray fields, magneto-motive forces, electromagnetic forces, and so on could be significantly reduced. The cold-pole scheme in the old design has been replaced with a warm-pole scheme.

New design of the sector magnet is now almost in its final stage. Winding of the main coil is scheduled to start in the autumn of 2001. The cool-down and magnet excitation is scheduled for the autumn of 2004.

\section{REFERENCES}

[1] Y. Yano et al., "RI Beam Factory Project at RIKEN", in this proceedings.

[2] A. Goto et al., "The K2500 Superconducting Ring Cyclotron of RIKEN RI Beam Factory - Overview and Status -", Proc. 15th Int. Conf. on Cyclotron and Their Applications, Caen, pp.148-155 (1998).

[3] S. Fujishima et al., "Design of the Injection and extraction Systems for the RIKEN SRC", in this proceedings.

[4] H. Okuno et al., "Design and Construction of the Superconducting Bending Magnet for the Injection System of the RIKEN SRC", in this proceedings. 